\title{
Temporomandibular Joint Disc Adhesion Without Mouth-Opening Limitation
}

\author{
Paulo S.F. Campos, PhD, * João B. Macedo Sobrinbo, PhD, $†$ \\ Iêda M.R. Crusoé-Rebello, PhD, $\neq$ Nilson Pena, PhD, $\downarrow$ \\ Janaina A. Dantas, MS, DDS, || Ana C.R. Mariz, MS, DDS, $₫$ and \\ Christiano Oliveira, DDS\#
}

We present a case of a 26-year-old female patient complaining of articular pain and clicking on the left side, caused by mastication, of 5 years' duration. Clinically, the patient presented with jaw-opening limitation, with deflection of the mandible to the right side without correction of the trajectory at the end of the jaw-opening movement. Imaging studies led to a diagnosis of bilateral disk adhesion (anchored disc phenomenon [ADP]) and slight limitation of the right condyle translation.

This case, likely the first to be reported in the literature, confirms that reciprocal clicking may occur in cases of ADP and that, contrary to the general concept, ADP without mouth-opening limitation can occasionally occur.

\section{Report of a Case}

A 26-year-old female patient was referred to a private clinic to undergo magnetic resonance imaging (MRI) of the

\footnotetext{
*Associate Professor, Department of Radiology, School of Dentistry, Federal University of Bahia, Bahia, Brazil.

†Adjunct Professor, Department of Anatomy, Metropolitan University Dental School, Bahia, Brazil.

‡Adjunct Professor, Department of Radiology, School of Dentistry, Federal University of Bahia, Bahia, Brazil.

SAdjunct Professor, Department of Radiology, School of Dentistry, Bahia State University, Bahia, Brazil.

||Postgraduate Student, Department of Radiology, Piracicaba Dental School, Campinas University, São Paulo, Brazil

ISubstitute Professor, Department of Radiology, School of Dentistry, Federal University of Bahia, Bahia, Brazil.

\#Postgraduate Student, Department of Radiology, School of Dentistry, Federal University of Bahia, Bahia, Brazil.

Address correspondence and reprint requests to Dr Flores Campos: Rua Guadalajara, no 841, apt 201, Ondina CEP 40.140-460, Salvador, Bahia, Brasil; e-mail: pflores@ufba.br ( 2008 American Association of Oral and Maxillofacial Surgeons 0278-2391/08/6603-0022 \$34.00/0 doi:10.1016/i.joms.2006.11.006
}

temporomandibular joint (TMJ). The patient complained of articular pain and clicking on only the left side, caused by mastication, of 5 years' duration. No history of relevant disease, local trauma, or TMJ surgery was reported. Clinically, the patient presented with jaw-opening limitation $(4.5 \mathrm{~cm}$ measured at the midline), with deflection of the mandible to the right side without correction of the trajectory at the end of the jaw-opening movement. The mandibular deflection was accompanied by a clinically audible click that was also heard upon jaw closing. There was no restriction of protrusion and lateral movements, and no articular sound was detected on auscultation during these movements.

The closed-mouth T1-weighted MRI image shown in Figure $1(\mathrm{TR}=400 \mathrm{~ms} ; \mathrm{TE}=13 \mathrm{~ms} ; \mathrm{NEX}=4 ; 14-\mathrm{cm}$ FOV; $256 \times 256 \times 2 \mathrm{~mm}$ thick [no gap]) reveals the posterior position of the condyle with the normal position of the disk in relation to the temporal component. The images show a similar condition on the left side. No bone alteration is exhibited on either side.

On the right side, the open-mouth images (Fig 2) reveal a slightly limited condyle translation and disc immobility, with the disc remaining in the same position in relation to the temporal component. This lack of disc movement is also seen on the left side, but there the condyle surmounts the anterior band of the disc and performs a normal translation.

The foregoing imaging features led to the diagnosis of bilateral disc adhesion and slight limitation of the right condyle translation.

\section{Discussion}

Even though anterior disc displacement without reduction is not a jaw-opening limiting factor for some patients, closed-lock TMJ is related to anterior disc displacement without reduction in many cases. ${ }^{1,2}$ In these cases, the nonrecaptured disc acts as a mechanical obstacle preventing free condyle translation, thereby restricting mouth opening. In addition, the displaced disc may become stuck on the articular eminence and permanently limit condyle translation. ${ }^{3}$

According to Kaminishi and Davis, ${ }^{4}$ disc adhesions occur most often in the upper joint space and can result from 2 mechanisms. First, synovitis may lead to the deposition of a layer of fibrin over the cells of the synovium instead of the normal hyaluronic acid layer. With evolution of the disease, this fibrin layer over the 

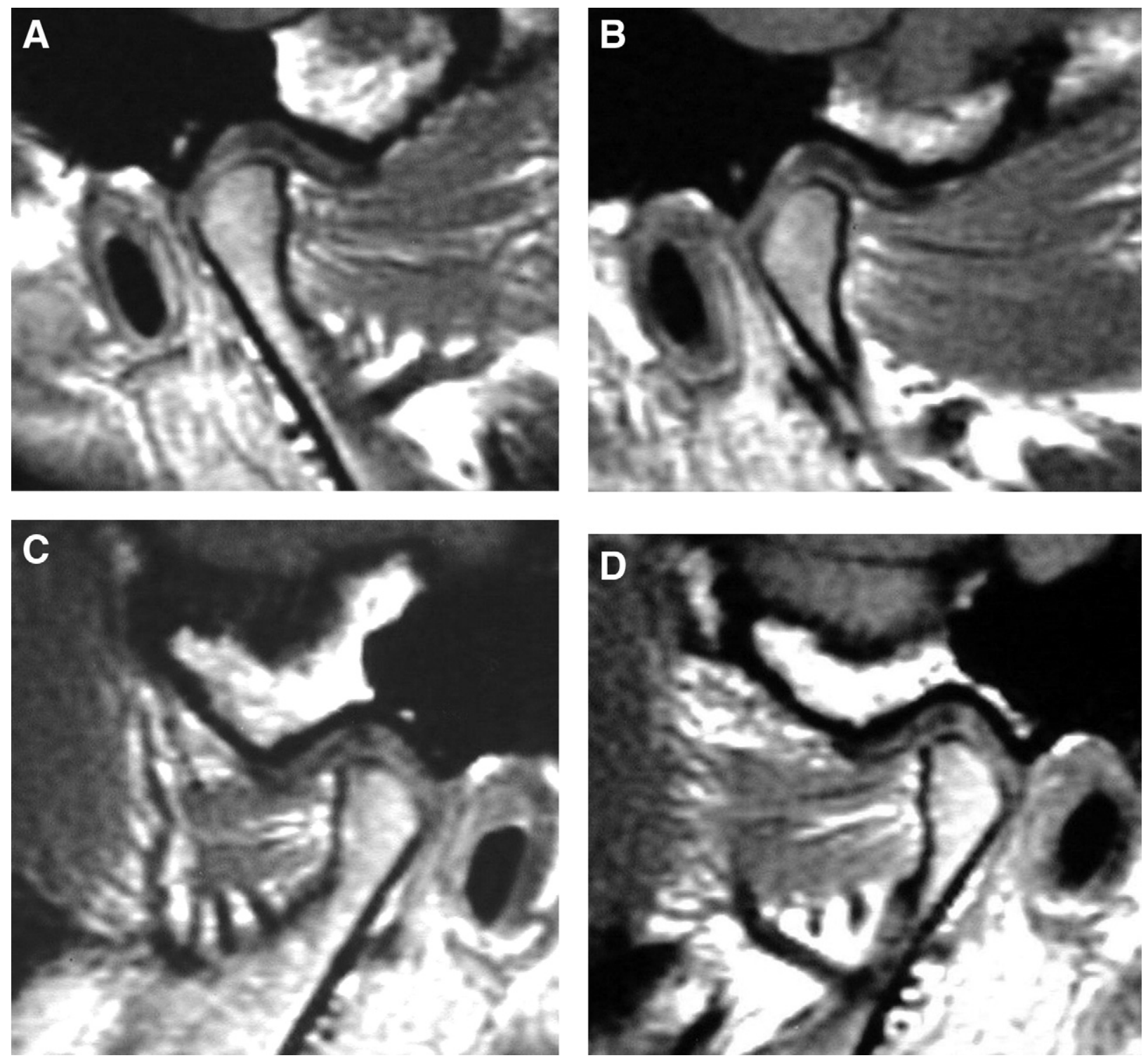

FIGURE 1. Closed-mouth parasagittal images of both sides displaying retropositioning of the condyle and normal position of the disc in relation to the temporal component.

Campos et al. TMJ Disc Adhesion Without Mouth-Opening Limitation. J Oral Maxillofac Surg 2008.

articular surfaces changes into fibrous tissue, producing disc adhesion. Second, hematomas, which are commonly found in the upper joint space, pass through a natural process of healing with capillary invasion and gradual transition to scar-like fibrous tissue, promoting disc adhesion.

Concerning the types of adhesion that can occur between the disc-retrodiscal tissue and the bone surface of the temporal component, Kaminishi and Davis, ${ }^{4}$ based on arthroscopic observations of anterior displaced discs, classified TMJ disc adhesions in order of increasing severity as fibrous bands, fibrosynovial bands, intracapsular fibrosis, discal osseous bands, partial pseudowall, intermittent pseudowall, fibrous pseudowall, and fibrosynovial pseudowall. In addi- tion, they described TMJ capsular fibrosis and stressed that all forms of pseudowall fall under the definition of fibrous ankylosis.

The adhesion also may occur with the disc in its normal position, determining mouth-opening limitation in the same way. In this condition, termed ADP, the patient displays a severe mouth-opening limitation (15 to $25 \mathrm{~mm}$ ) and rarely refers to a history of clicks, in contrast to anterior disc displacement without reduction, in which the patient displays a less severe mouth-opening limitation (30 to $45 \mathrm{~mm}$ ) and reports past clicks. ${ }^{5}$

Nitzan and Etsion ${ }^{5}$ suggested that a normal lubrication system consisting of hyaluronic acid and protecting phospholipids enables movement of the articular 

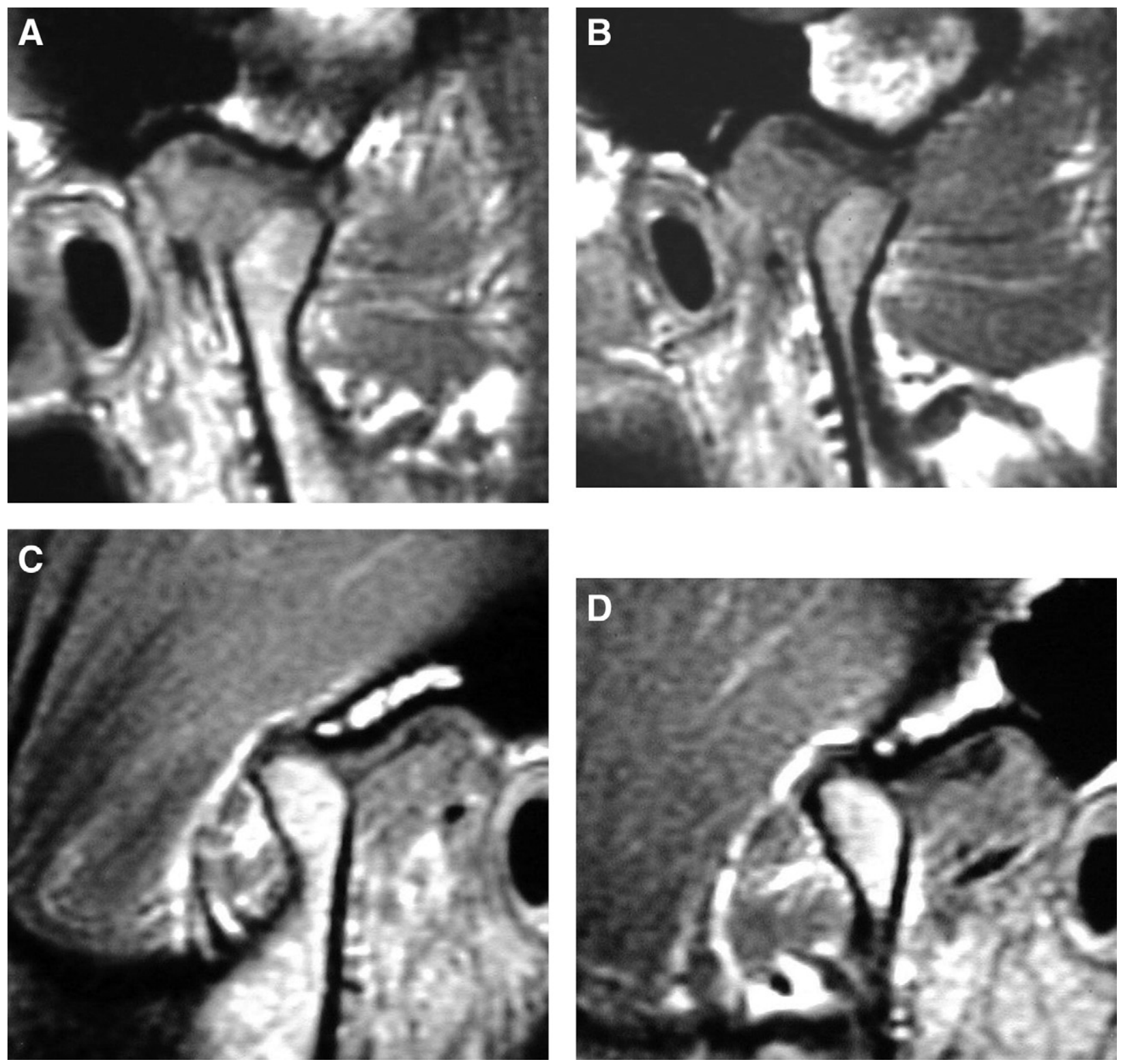

FIGURE 2. Open-mouth parasagittal images showing, on the right, slight limitation of the condyle translation, and, on the left, normal condyle translation. The disc is in the same position on both sides.

Campos et al. TMJ Disc Adhesion Without Mouth-Opening Limitation. J Oral Maxillofac Surg 2008.

disk. In cases of joint overload, however, uncontrolled production of reactive oxygen species may occur, causing degradation of the hyaluronic acid and subsequent exposure of the phospholipids to lysis by phospholipase $\mathrm{A}^{2}$. Thus, the denuded articular surfaces, with high surface energy, become strongly adherent when placed in contact with one another. The presence of a thin film of fluid between the articulating surfaces may further increase the adhesion potential. Consequently, the articular disc may become stuck to the temporal component in its normal position; this condition is termed ADP.

In our patient, the imaging features allowed us to classify the disc adhesion as ADP. On the other hand, its association with reciprocal clicking and normal translation of the left condyle, along with slight limitation of the right condyle translation, contradict the ADP characteristics stated by Nitzan and Etsion: severe mouthopening limitation (15 to $25 \mathrm{~mm}$ ) and no history of clicks in most cases. ${ }^{4}$ Clearly, restriction of the condyle translation is not always present in ADP; in some cases, the condyle can surmount the anterior band of the disc and perform a normal translation. The reciprocal clicking will always occur when the condyle passes over the anterior band of the articular disc, at the end of the jaw-opening movement and during the jaw-closing movement.

In conclusion, to the best of our knowledge, this is the first reported case of closed-lock TMJ associated 
with reciprocal clicking and normal translation of the condyle. Thus, we can assert that reciprocal clicking may occur in cases of ADP, and, in contrast to the general concept, ADP can occasionally occur without severe mouth-opening limitation.

\section{References}

1. Katzberg RW, Westesson P-L: Diagnosis of the Temporomandibular Joint. Philadelphia, Saunders, 1994, pp 25-70
2. Mariz ACR, Campos PSF, Sarmento VA, et al: Assessment of disk displacements of the temporomandibular joint. Brazil Oral Res 19:63, 2005

3. Rao VM, Liem MD, Farole A, et al: Elusive "stuck" disk in the temporomandibular joint: Diagnosis with MR imaging. Radiology 189:823, 1993

4. Kaminishi RM, Davis CL: Temporomandibular joint arthroscopic observations of superior space adhesions. Oral Maxillofac Surg Clin North Am 1:103, 1989

5. Nitzan DW, Etsion I: Adhesive force: The underlying cause of the disc anchorage to the fossa and/or eminence in the temporomandibular joint. A new concept. Int J Oral Maxillofac Surg 31:94, 2002

\title{
Management of the Oral Surgery Patient Diagnosed With Epidermolysis Bullosa: Report of 3 Cases and Review of the Literature
}

\author{
Franci Stavropoulos, DDS* and \\ Shelly Abramowicz, DMD, MPH†
}

Epidermolysis bullosa (EB) is the name given to a group of rare, genetically determined diseases characterized by cutaneous and mucosal blistering associated with occasional subsequent scarring secondary to minor trauma. It is divided into 3 major types by histological findings, and includes approximately 23 variants, manifested by a spectrum of clinical presentations. ${ }^{1}$ Occasionally, the oral and maxillofacial surgeon will be called upon to treat patients with $\mathrm{EB}$ and should therefore be aware of specific treatment modifications. We present a review of the literature with examples of 3 cases treated at the University of Florida Department of Oral and Maxillofacial Surgery. These cases demonstrate the extensive spectrum of this disease and the treatment adjustments necessary

Received from the Department of Oral and Maxillofacial Surgery and Diagnostic Sciences, University of Florida College of Dentistry,

Gainesville, FL.

*Associate Professor.

tResident.

Address correspondence and reprint requests to Dr Stavropoulos: Department of Oral \& Maxillofacial Surgery \& Diagnostic Sciences, JHMHC, PO Box 100416, 1600 SW Archer Road D7-6, Gainesville, FL 32610-0416; e-mail: fstavrop@dental.ufl.edu (1) 2008 American Association of Oral and Maxillofacial Surgeons 0278-2391/08/6603-0023\$34.00/0 doi:10.1016/i.joms.2007.06.672 to minimize trauma to the soft tissues of EB patients while providing adequate and thorough anesthetic and surgical care.

\section{Report of Cases}

\section{CASE 1}

A 26-year-old female patient, diagnosed with dystrophic EB at birth, was referred by her general dentist with a chief complaint of bilateral jaw pain, oral ulcerations, and limited mouth opening due to oral scar bands. She reported nocturnal grinding of her teeth. In addition, she reported daily gum chewing which exacerbated the ulcerations in her mouth. Because of her disease causing esophageal strictures which lead to dysphagia, she had had dilations of her esophageal strictures at 2 different occasions. She regularly used over-the-counter pain medications to control her pain. She had only 1 hospitalization episode for management of her EB. Her past medical history was otherwise unremarkable. On physical examination, the patient's fingers were normal, but her toenail beds were noted to be dystrophic bilaterally. On her right foot a contracture was present extending to her midcalf where the patient reported a few blistering episodes secondary to repetitive trauma. Her extraoral evaluation revealed a maximal interincisal opening of $30 \mathrm{~mm}$ with small, fibrous scar bands bilaterally at the commisures of the lips, likely causing progressing microstomia. Her intraoral examination revealed a high arched palate, ankyloglossia, generalized ulcerative gingivitis, and multiple carious molars. The buccal mucosa exhibited horizontal scar bands bilaterally (Fig 1), mild ulcerations, and a Mallampati classification of I. A panorex radiograph was significant for 